\title{
Reactions of New $N$-(2,2-Dichloro-1-cyanoethenyl)amides with Aliphatic Amines
}

\author{
O. V. Shablykin ${ }^{a}$, S. A. Chumachenko ${ }^{a}$, and V. S. Brovarets ${ }^{a, *}$ \\ ${ }^{a}$ V.P. Kukhar Institute of Bioorganic Chemistry and Petrochemistry, National Academy of Science of Ukraine, \\ Kyiv, 02094 Ukraine \\ *e-mail: brovarets@bpci.kiev.ua
}

Received July 23, 2021; revised July 23, 2021; accepted August 9, 2021

\begin{abstract}
The reaction of the newly synthesized N-(2,2-dichloro-1-cyanoethenyl)prop-2-enamide and 4-chloro$\mathrm{N}$-(2,2-dichloro-1-cyanoethenyl)butanamide with methylamine or dimethylamine gave rise to previously unknown 5-amino-1,3-oxazole-4-carbonitriles. In the case of the reaction of $\mathrm{N}$-(2,2-dichloro-1-cyanoethenyl)amides with ethylenediamine diacetate, new (Z)-2,3,5,6,7,8-hexahydro-7-oxo- $1 \mathrm{H}$-imidazo[1,2- $a][1,4]$ diazepine-9-carbonitrile and 4-chloro- $N$-(cyano(imidazolidin-2-ylidene)methyl)butanamide were obtained.
\end{abstract}

Keywords: cyclization, 5-alkylamino-2-aminoalkyl-1,3-oxazole-4-carbonitrile, (Z)-2,3,5,6,7,8-hexahydro7-oxo-1 $H$-imidazo[1,2-a][1,4]diazepine-9-carbonitrile, 2-amino-3,3-dichloroacrylonitrile acyl derivatives

DOI: $10.1134 / \mathrm{S} 1070363221090012$

Previously, we have shown that substituted 2-acylamino-3,3-dichloroacrylonitriles can be used to produce various heterocycles such as thiophene [1], hydantoin [2], and indole derivatives [3]. However, most reactions of 2-acylamino-3,3-dichloroacrylonitriles with amines provide oxazole derivatives [4-6]. In this case, depending on the nature of the acyl residue, the resulting reaction products can enter into further transformations with the retention of the oxazole ring [6-8], or with its destruction and the formation of recyclization products [9].

Herein, we reported the synthesis of previously unknown $N$-(2,2-dichloro-1-cyanoethenyl)prop-2enamide 2 and $N$-(2,2-dichloro-1-cyanoethenyl)-4chlorobutylamide 3 (Scheme 1) starting from available 2-amino-3,3-dichloroacrylonitrile 1 [10] and studied their reactions with some aliphatic amines (methylamine, dimethylamine, and ethylenediamine). Thus, reaction of $N$-(2,2-dichloro-1-cyanoethenyl)prop-2-enamide 2 with an excess of methylamine or dimethylamine proceeds through oxazole cyclization with the simultaneous addition of the amine residue to the double bond. As a result, 5-alkylamino-2-aminoethyl-1,3-oxazole-4carbonitriles $\mathbf{4 a}$ and $\mathbf{4 b}$ were prepared (Scheme 2). It should be noted that oxazole 4a was isolated in a yield of only $20 \%$. According to the data of liquid chromatography-mass spectra, compound $\mathbf{5}$ is probably also present in the reaction mixture; however, we failed to isolate it in an individual state.

Scheme 1.

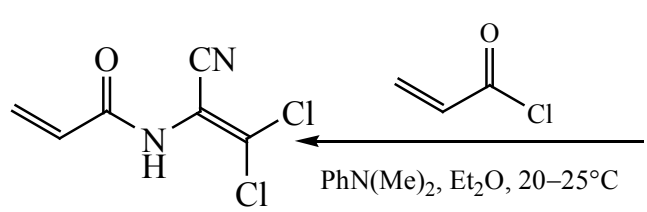

2<smiles>N#CC(N)=C(Cl)Cl</smiles>

1<smiles>N#CC(NC(=O)CCCCl)=C(Cl)Cl</smiles>

3 
Scheme 2.<smiles>[R2]NCCc1nc(C#N)c([Y17])o1</smiles>

4a, $4 b$<smiles>CNC1=C(C#N)NC(=O)CCN1C</smiles>

$5\left(\mathrm{R}^{1} \mathrm{R}^{2} \mathrm{NH}=\mathrm{MeNH}_{2}\right)$ $\mathrm{H}_{2} \mathrm{~N}\left(\mathrm{CH}_{2}\right)_{2} \mathrm{NH}_{2} \cdot 2 \mathrm{AcOH}$
$20-25^{\circ} \mathrm{C}, 48 \mathrm{~h}$<smiles>CC(C)NC(=O)CCN1CCNC(Cl)=C1C#N</smiles>
$\mathrm{R}^{1} \mathrm{R}^{2} \mathrm{~N}=\mathrm{MeNH}(\mathbf{4 a}),(\mathrm{Me})_{2} \mathrm{~N}(\mathbf{4 b})$.

Scheme 3.

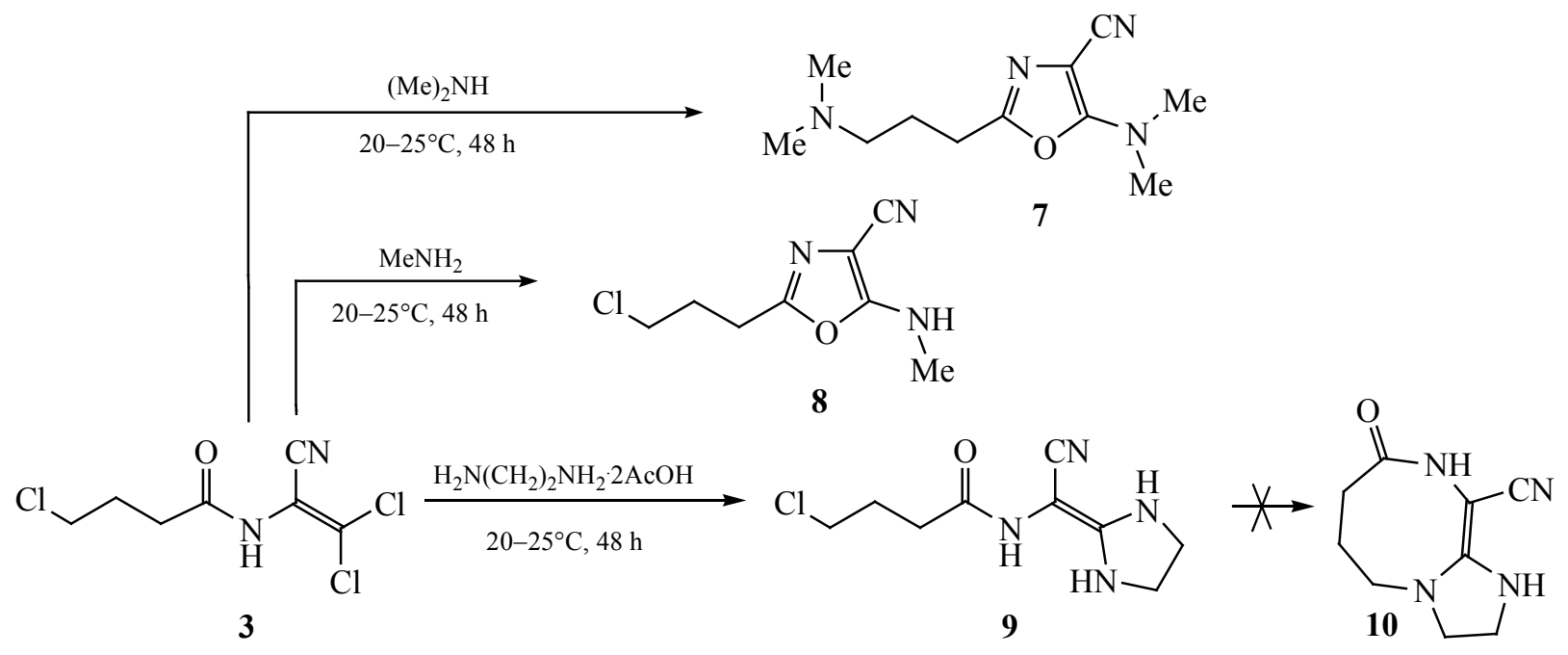

The reaction of dichloroacrylonitrile $\mathbf{2}$ with ethylenediamine diacetate resulted in the formation of $(Z)$ 2,3,5,6,7,8-hexahydro-7-oxo- $1 H$-imidazo[1,2- $a][1,4]$ diazepine-9-carbonitrile 6. A plausible mechanism for the formation of compound $\mathbf{6}$ includes the formation of intermediates $\mathbf{A}$ and $\mathbf{B}$ (Scheme 2).

The reaction of $\mathrm{N}$-(2,2-dichloro-1-cyanoethenyl)4-chlorobutylamide 3 with dimethylamine proceeded similarly to compound 2 . In this case, 5-(dimethylamino)2-[3-(dimethylamino)propyl]-1,3-oxazole-4-carbonitrile 7 was formed in high yield (Scheme 3). Substituted 5-amino-1,3-oxazole-4-carbonitrile 8 containing a chloropropyl residue in the second position of the ring was isolated, when using an excess of methylamine. Probably, the substitution of the chlorine atom in the chloropropyl residue was not observed due to the lower nucleophilicity of methylamine as compared to dimethylamine. The 
Scheme 4.

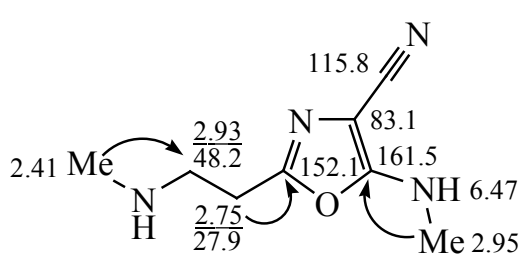

$4 a$

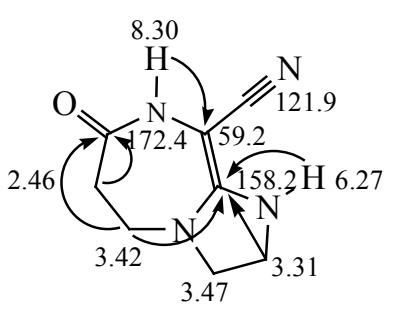

6 reaction of ethylenediamine diacetate with nitrile $\mathbf{3}$ led to the formation of ketenaminal $\mathbf{9}$, which was isolated in the acetate form. Attempts to carry out targeted intramolecular alkylation of the ketenaminal moiety with a chloropropyl residue in order to obtain a bicyclic structure $\mathbf{1 0}$ were unsuccessful.

Structure and purity of the obtained compounds were proved by the liquid chromatography-mass spectrometry, IR spectroscopy, elemental analysis, and NMR spectroscopy methods. Structure of compounds 4a and 6 was unambiguously confirmed by means of 2D NMR techniques (COSY, HSQC, HMBC) (Scheme 4). The presence in the ${ }^{1} \mathrm{H}$ NMR spectra of signals of aliphatic protons and NH-groups, as well as their multiplicity, make it possible to determine the corresponding fragments in the molecule of compound 4a. The protons of the methylene $(2.75 \mathrm{ppm})$ and methyl $(2.95 \mathrm{ppm})$ groups in the HMBC spectrum correlate with the $\mathrm{C}^{2}$ and $\mathrm{C}^{5}$ carbon atoms $(152.1,162.5 \mathrm{ppm})$, which is characteristic of the 5-aminooxazole fragment.

Structure of compound $\mathbf{6}$ is confirmed by the presence of the signal of an $\mathrm{NH}$ proton at $8.30 \mathrm{ppm}$ in the ${ }^{1} \mathrm{H}$ NMR spectrum. The fact that the NH group is directly bonded to the carbon atom at the nitrile group (59.2 ppm) is confirmed by HMBC correlations. Also, the protons of the $\mathrm{CH}_{2}$ groups of the diazepine ring in the HMBC spectrum correlate with the carbon atom of the carbonyl group (172.37 ppm). The interaction of protons of two methylene groups (3.31 and $3.42 \mathrm{ppm}$ ), as well as one NH-proton with a carbon atom at a double bond $(158.2 \mathrm{ppm})$ indicates the formation of $(Z)-2,3,5,6,7,8$ hexahydro-7-oxo- $1 H$-imidazo[1,2- $a][1,4]$ diazepine ring.

In summary, we first studied the reactions of $N$-(2,2-dichloro-1-cyanoethenyl)prop-2-enamide and $N$-(2,2-dichloro-1-cyanoethenyl)-4-chlorobutylamide with some aliphatic amines. At the same time, new representatives of 5-amino-1,3-oxazole-4-carbonitriles were obtained, as well as $(Z)-2,3,5,6,7,8$-hexahydro7-oxo- $1 H$-imidazo[1,2- $a][1,4]$ diazepine-9-carbonitrile and 4-chloro- $N$-[cyano(imidazolidin-2-ylidene)methyl]butanamide in the case of the reaction with ethylenediamine.

\section{EXPERIMENTAL}

IR spectra were recorded on a Vertex-70 spectrometer from $\mathrm{KBr}$ pellets. ${ }^{1} \mathrm{H}$ and ${ }^{13} \mathrm{C}$ NMR spectra were taken on a Bruker AVANCE DRX-500 instrument (500 and $125 \mathrm{MHz}$, respectively) from DMSO- $d_{6}$ or $\mathrm{CDCl}_{3}$ solution. Chromato-mass spectra were recorded using a liquid chromatography-mass spectrometry system on an Agilent 1100 Series high performance liquid chromatograph equipped with an Agilent LC IMSD SL mass selective detector diode array. Chromatographymass analysis parameters: column Zorbax SB-C18 $1.8 \mu \mathrm{m}$ $4.6 \times 15 \mathrm{~mm}(\mathrm{PN} 821975-932)$; solvents: A, $\mathrm{MeCN}-\mathrm{H}_{2} \mathrm{O}$, $95: 5,0.1 \%$ TFA; $\mathrm{B}, 0.1 \%$ aqueous TFA; eluent flow $3 \mathrm{~mL} / \mathrm{min}$; injection volume $1 \mu \mathrm{L}$; UV detectors: 215 , $254,285 \mathrm{~nm}$; ionization method was atmospheric pressure chemical ionization (APCI), scanning range $\mathrm{m} / \mathrm{z} 80$ $1000 \mathrm{Da}$. Elemental analysis was performed in the analytical laboratory of the V.P. Kukhar Institute of Bioorganic Chemistry and Petrochemistry of the National Academy of Sciences of Ukraine. The content of carbon and hydrogen was determined by the Pregl gravimetric method, nitrogen - by the Dumas gas-metric micromethod, and chlorine-by the Schöniger titrimetric method [11]. Melting points were measured on a FisherJohns apparatus. The reaction progress and the purity of the obtained compounds were monitored by thin layer chromatography on Macherey-Nagel ALUGRAM Xtra SIL G/UV254 plates in the chloroform-methanol system $(10: 0.2)$.

2-Amino-3,3-dichloroacrylonitrile $\mathbf{1}$ as well as acrylic and 4-chlorobutanoic acid chlorides are commercial products. 
$N$-(2,2 -Dichloro-1-cyanoethenyl)prop-2 enamide (2) and $N$-(2,2-dichloro-1-cyanoethenyl)-4chlorobutylamide (3) were obtained by the previously described method [6]. To a solution of $13.7 \mathrm{~g}(100 \mathrm{mmol})$ of 2-amino-3,3-dichloroacrylonitrile 1 and $12.1 \mathrm{~g}$ (100 mmol) of $N, N$-dimethylaniline in $100 \mathrm{~mL}$ of diethyl ether with vigorous stirring was added dropwise the corresponding acid chloride $(100 \mathrm{mmol})$ at $20-25^{\circ} \mathrm{C}$. The resulting mixture was stirred for $12 \mathrm{~h}$, then $200 \mathrm{~mL}$ of a water-hexane mixture $(1: 1)$ was added. A precipitate formed in the two-phase system was filtered off, washed with $50 \mathrm{~mL}$ of a hexane-diethyl ether mixture $(1: 1)$, and dried in a vacuum. Compounds $\mathbf{2}, \mathbf{3}$ were used for further transformations without additional purification.

$\mathrm{N}$-(2,2-Dichloro-1-cyanoethenyl)prop-2-enamide (2). Yield $75 \%$, mp $140-142^{\circ} \mathrm{C}$. IR spectrum, $\mathrm{v}, \mathrm{cm}^{-1}$ : 1495, 1599, 1630, 1670, $2230(\mathrm{CN}), 3033-3238(\mathrm{NH})$. ${ }^{1} \mathrm{H}$ NMR spectrum (DMSO- $\left.d_{6}\right), \delta$, ppm: $2.21 \mathrm{~d}(1 \mathrm{H}$, $\left.\mathrm{CH}_{2}=\mathrm{CH},{ }^{3} J_{\mathrm{HH}} 10.0 \mathrm{~Hz}\right), 6.29-6.46 \mathrm{~m}\left(2 \mathrm{H}, \mathrm{CH}_{2}=\mathrm{CH}\right)$, $10.48 \mathrm{~s}(1 \mathrm{H}, \mathrm{NH}) .{ }^{13} \mathrm{C}$ NMR spectrum $\left(\mathrm{DMSO}-d_{6}\right), \delta_{\mathrm{C}}$, ppm: 109.2, 110.8, 127.5, 128.2, 129.8, $161.8(\mathrm{C}=\mathrm{O})$. Mass spectrum, $m / z$ : $191[M+\mathrm{H}]^{+}$. Found, \%: C 37.17; $\mathrm{H}$ 2.47; $\mathrm{Cl} 37.03 ; \mathrm{N} 14.40 . \mathrm{C}_{6} \mathrm{H}_{4} \mathrm{Cl}_{2} \mathrm{~N}_{2} \mathrm{O}$. Calculated, \%: C $37.73 ; \mathrm{H} 2.11 ; \mathrm{Cl} 37.12 ; \mathrm{N} 14.67$.

$\mathrm{N}$-(2,2-Dichloro-1-cyanoethenyl)-4-chlorobutylamide (3). Yield $80 \%, \mathrm{mp} 97-99^{\circ} \mathrm{C}$. IR spectrum, $v, \mathrm{~cm}^{-1}$ : 1443, 1497, 1601, 1671, 2233 (CN), 3085-3284 (NH). ${ }^{1} \mathrm{HNMR}$ spectrum(DMSO- $d_{6}$ ), $\delta$, ppm: $1.83-1.90 \mathrm{~m}$ (2H, $\left.\mathrm{CH}_{2}\right), 2.35-2.38 \mathrm{~m}\left(2 \mathrm{H}, \mathrm{CH}_{2}+\mathrm{DMSO}\right), 3.53-3.56 \mathrm{~m}$ $\left(2 \mathrm{H}, \mathrm{CH}_{2}+\mathrm{H}_{2} \mathrm{O}\right), 10.20 \mathrm{~s}(1 \mathrm{H}, \mathrm{NH}) .{ }^{1} \mathrm{H}$ NMR spectrum $\left(\mathrm{CDCl}_{3}\right), \delta$, ppm: 2.16-2.22 m $\left(2 \mathrm{H}, \mathrm{CH}_{2}\right), 2.59 \mathrm{t}(2 \mathrm{H}$, $\left.\mathrm{CH}_{2},{ }^{3} J_{\mathrm{HH}} 7.1 \mathrm{~Hz}\right), 3.66 \mathrm{t}\left(2 \mathrm{H}, \mathrm{CH}_{2},{ }^{3} J_{\mathrm{HH}} 6.1 \mathrm{~Hz}\right)$, 7.09 br. s $(1 \mathrm{H}, \mathrm{NH}) .{ }^{13} \mathrm{C}$ NMR spectrum (DMSO- $d_{6}$ ), $\delta_{\mathrm{C}}$, ppm: $25.8\left(\mathrm{CH}_{2}\right), 30.2\left(\mathrm{CH}_{2}\right), 43.1\left(\mathrm{CH}_{2}\right), 109.7$, $111.3,129.9,169.7(\mathrm{C}=\mathrm{O})$. Mass spectrum, $m / z: 241$ $[M+\mathrm{H}]^{+}$. Found, \%: C 34.77; H 3.15; Cl 43.71; N 11.36 . $\mathrm{C}_{7} \mathrm{H}_{7} \mathrm{Cl}_{3} \mathrm{~N}_{2} \mathrm{O}$. Calculated, \%: C 34.81; H 2.92; $\mathrm{Cl} 44.04$; $\mathrm{N} 11.60$.

5-Alkylamino-2-aminoethyl-1,3-oxazole-4-carbonitriles (4a, 4b). To a solution of $10 \mathrm{mmol}$ of compound 2 in $20 \mathrm{~mL}$ of methanol with vigorous stirring was added $10 \mathrm{~mL}$ of a $40 \%$ aqueous solution of dimethylamine or $20 \mathrm{~mL}$ of a $20 \%$ methanol solution of methylamine at $20-25^{\circ} \mathrm{C}$. The solution was stirred for $48 \mathrm{~h}$, the excess of the corresponding amine, and the solvent was removed in vacuum at $40^{\circ} \mathrm{C}$. A solution of $6.9 \mathrm{~g}(50 \mathrm{mmol})$ of $\mathrm{K}_{2} \mathrm{CO}_{3}$ in $20 \mathrm{~mL}$ of water was added to the residue, and compound 4 was extracted with methylene chloride
$(3 \times 30 \mathrm{~mL})$. The extract was washed with water $(4 \times 5 \mathrm{~mL})$ and dried with $\mathrm{Na}_{2} \mathrm{SO}_{4}$, the solvent was removed in vacuum. The obtained compounds were purified by flash chromatography, using a mixture $\mathrm{CH}_{2} \mathrm{Cl}_{2}$-methanol as eluent.

5-(Methylamino)-2-[2-(methylamino)ethyl]-1,3oxazole-4-carbonitrile (4a). Yield 20\%, mp 107-109 ${ }^{\circ} \mathrm{C}$. IR spectrum, $v, \mathrm{~cm}^{-1}: 1473,1489,1604,1679,2198(\mathrm{CN})$, 3056-3263 (NH). ${ }^{1} \mathrm{H}$ NMR spectrum $\left(\mathrm{CDCl}_{3}\right), \delta$, ppm: $2.41 \mathrm{~s}\left(3 \mathrm{H}, \mathrm{CH}_{3}\right), 2.75 \mathrm{t}\left(2 \mathrm{H}, \mathrm{CH}_{2},{ }^{3} J_{\mathrm{HH}} 7.5 \mathrm{~Hz}\right), 2.93 \mathrm{t}$ $\left(2 \mathrm{H}, \mathrm{CH}_{2},{ }^{3} J_{\mathrm{HH}} 7.5 \mathrm{~Hz}\right), 2.95 \mathrm{~s}\left(3 \mathrm{H}, \mathrm{CH}_{3}\right), 6.47$ br. s $(1 \mathrm{H}$, $\mathrm{NH}) .{ }^{13} \mathrm{C} \mathrm{NMR}$ spectrum $\left(\mathrm{CDCl}_{3}\right), \delta_{\mathrm{C}}, \mathrm{ppm}: 27.9\left(\mathrm{CH}_{2}\right)$, $29.8\left(\mathrm{CH}_{3}\right), 35.9\left(\mathrm{CH}_{3}\right), 48.2\left(\mathrm{CH}_{2}\right), 83.2\left(\mathrm{C}^{4}\right.$, oxazole), $115.8(\mathrm{CN}), 152.1$ ( $\mathrm{C}^{2}$, oxazole), 161.5 ( $\mathrm{C}^{5}$, oxazole). Mass spectrum, $m / z$ : $181[M+\mathrm{H}]^{+}$. Found, \%: C 53.27; H 6.89; N 30.78. $\mathrm{C}_{8} \mathrm{H}_{12} \mathrm{~N}_{4} \mathrm{O}$. Calculated, \%: C 53.32; H $6.71 ; \mathrm{N} 31.09$.

5-(Dimethylamino)-2-[2-(dimethylamino)ethyl]1,3-oxazole-4-carbonitrile (4b). Yield 90\%, mp 38$40^{\circ} \mathrm{C}$. IR spectrum, $v, \mathrm{~cm}^{-1}: 1464,1604,1654 \mathrm{sh}, 2204$ (CN). ${ }^{1} \mathrm{H}$ NMR spectrum $\left(\mathrm{CDCl}_{3}\right), \delta$, ppm: $1.97 \mathrm{~s}(6 \mathrm{H}$, $\left.\mathrm{CH}_{3}\right), 2.36 \mathrm{t}\left(2 \mathrm{H}, \mathrm{CH}_{2},{ }^{3} \mathrm{~J}_{\mathrm{HH}} 7.0 \mathrm{~Hz}\right), 2.45 \mathrm{t}\left(2 \mathrm{H}, \mathrm{CH}_{2},{ }^{3} \mathrm{~J}_{\mathrm{HH}}\right.$ $7.0 \mathrm{~Hz}), 2.83 \mathrm{~s}\left(6 \mathrm{H}, \mathrm{CH}_{3}\right) \cdot{ }^{13} \mathrm{C}$ NMR spectrum $\left(\mathrm{CDCl}_{3}\right)$, $\delta_{\mathrm{C}}$, ppm: $24.9\left(\mathrm{CH}_{2}\right), 37.4,43.8\left(2 \mathrm{CH}_{3}\right), 54.6,83.1\left(\mathrm{C}^{4}\right.$, oxazole), $111.5(\mathrm{CN}), 151.3\left(\mathrm{C}^{2}\right.$, oxazole $), 160.3\left(\mathrm{C}^{5}\right.$, oxazole). Mass spectrum, $m / z: 209[M+\mathrm{H}]^{+}$. Found, \%: C 57.81; H 7.50; N 27.32. $\mathrm{C}_{10} \mathrm{H}_{16} \mathrm{~N}_{4} \mathrm{O}$. Calculated, \%: C $57.67 ; \mathrm{H} 7.74 ; \mathrm{N} 26.90$.

(Z)-2,3,5,6,7,8-Hexahydro-7-oxo-1 $H$-imidazo[1,2-a][1,4]diazepine-9-carbonitrile (6). To a solution of $7.2 \mathrm{~g}(40 \mathrm{mmol})$ of ethylenediamine diacetate in $20 \mathrm{~mL}$ of methanol with vigorous stirring was added in portions ( $0.2 \mathrm{~g}$ each) $1.9 \mathrm{~g}(10 \mathrm{mmol})$ of compound 2 at $20-25^{\circ} \mathrm{C}$. The reaction mixture was stirred for $48 \mathrm{~h}$, then the precipitate was filtered off, washed with water $(2 \times$ $5 \mathrm{~mL}$ ), and dried at $50^{\circ} \mathrm{C}$. Compound 6 was analyzed without further purification. Yield $20 \%$, dp $190-195^{\circ} \mathrm{C}$. IR spectrum, $v, \mathrm{~cm}^{-1}: 1432,1480,1503,1608,1654,2172$ (CN), 3044-3243 (NH). ${ }^{1} \mathrm{H}$ NMR spectrum (DMSO- $d_{6}$ ), $\delta$, ppm: $2.46 \mathrm{t}\left(2 \mathrm{H}, \mathrm{CH}_{2},{ }^{3} J_{\mathrm{HH}} 5.4 \mathrm{~Hz}\right), 3.29-3.34$ br. $\mathrm{m}$ $\left(2 \mathrm{H}, \mathrm{CH}_{2}\right), 3.42 \mathrm{t}\left(2 \mathrm{H}, \mathrm{CH}_{2},{ }^{3} \mathrm{~J}_{\mathrm{HH}} 5.4 \mathrm{~Hz}\right), 3.47 \mathrm{t}\left(2 \mathrm{H}, \mathrm{CH}_{2}\right.$, $\left.{ }^{3} J_{\mathrm{HH}} 8.1 \mathrm{~Hz}\right), 6.27 \mathrm{~s}(1 \mathrm{H}, \mathrm{NH}), 8.30 \mathrm{~s}(1 \mathrm{H}, \mathrm{NH}) .{ }^{13} \mathrm{C} \mathrm{NMR}$ spectrum $\left(\mathrm{DMSO}-d_{6}\right), \delta_{\mathrm{C}}$, ppm: $35.2\left(\mathrm{CH}_{2}\right), 42.0\left(\mathrm{CH}_{2}\right)$, $49.0\left(\mathrm{CH}_{2}\right), 53.5\left(\mathrm{CH}_{2}\right), 59.2\left[\mathrm{C}=\mathrm{C}\left(\mathrm{NCH}_{2}\right)_{2}\right], 121.9(\mathrm{CN})$, $158.2\left[\mathrm{C}=\underline{\mathrm{C}}\left(\mathrm{NCH}_{2}\right)_{2}\right], 172.4(\mathrm{C}=\mathrm{O})$. Mass spectrum, $m / z: 179[M+\mathrm{H}]^{+}$. Found, \%: C 54.15; H 5.79; N 31.76 . $\mathrm{C}_{8} \mathrm{H}_{10} \mathrm{~N}_{4} \mathrm{O}$. Calculated, \%: C 53.92; H 5.66; N 31.44 . 
5-(Dimethylamino)-2-[3-(dimethylamino)propyl]1,3-oxazole-4-carbonitrile (7). To a solution of $10 \mathrm{mmol}$ of compound 3 in $20 \mathrm{~mL}$ of methanol with vigorous stirring was added $10 \mathrm{~mL}$ of a $40 \%$ aqueous solution of dimethylamine at $20-25^{\circ} \mathrm{C}$. The solution was stirred for $48 \mathrm{~h}$, an excess of amine and the solvent were removed in vacuum at $40^{\circ} \mathrm{C}$. A solution of $6.9 \mathrm{~g}(50 \mathrm{mmol})$ of $\mathrm{K}_{2} \mathrm{CO}_{3}$ in $20 \mathrm{~mL}$ of water was added to the residue and extracted with methylene chloride $(3 \times 30 \mathrm{~mL})$. The extract was washed with water $(4 \times 5 \mathrm{~mL})$ and dried with $\mathrm{Na}_{2} \mathrm{SO}_{4}$, the solvent was removed in vacuum. Compound 7 was purified by flash chromatography eluting with $\mathrm{CH}_{2} \mathrm{Cl}_{2}$-methanol mixture. Yield 70\%, oil. IR spectrum, $v, \mathrm{~cm}^{-1}:$ 1444, 1600, 1641, $2207(\mathrm{CN}) .{ }^{1} \mathrm{H}$ NMR spectrum $\left(\mathrm{CDCl}_{3}\right), \delta$, ppm: $1.80-1.85 \mathrm{~m}\left(2 \mathrm{H}, \mathrm{CH}_{2}\right), 2.20 \mathrm{~s}(6 \mathrm{H}$, $\left.\mathrm{CH}_{3}\right), 2.30$ t $\left(2 \mathrm{H}, \mathrm{CH}_{2},{ }^{3} J_{\mathrm{HH}} 7.0 \mathrm{~Hz}\right), 2.62 \mathrm{t}\left(2 \mathrm{H}, \mathrm{CH}_{2}\right.$, $\left.{ }^{3} J_{\mathrm{HH}} 7.5 \mathrm{~Hz}\right), 3.11 \mathrm{~s}\left(6 \mathrm{H}, \mathrm{CH}_{3}\right) .{ }^{13} \mathrm{C}$ NMR spectrum $\left(\mathrm{CDCl}_{3}\right), \delta_{\mathrm{C}}, \mathrm{ppm}: 22.3\left(\mathrm{CH}_{2}\right), 23.3\left(\mathrm{CH}_{2}\right), 36.7\left(2 \mathrm{CH}_{3}\right)$, $43.3\left(2 \mathrm{CH}_{3}\right), 56.5\left(\mathrm{CH}_{2}\right), 82.2\left(\mathrm{C}^{4}\right.$, oxazole $), 114.9(\mathrm{CN})$, $152.1\left(\mathrm{C}^{2}\right.$, oxazole $), 159.6\left(\mathrm{C}^{5}\right.$, oxazole $)$. Mass spectrum, $m / z: 223[M+\mathrm{H}]^{+}$. Found, \%: C 59.47; H 8.42; N 25.59 . $\mathrm{C}_{11} \mathrm{H}_{18} \mathrm{~N}_{4} \mathrm{O}$. Calculated, \%: C 59.44; H 8.16; N 25.20.

5-(Methylamino)-2-(3-chloropropyl)-1,3-oxazole4-carbonitrile (8) was prepared similarly to oxazole 7 from compound 3 and $20 \mathrm{~mL}$ of a $20 \%$ methanol solution of methylamine. Yield $70 \%, \mathrm{mp} 69-71^{\circ} \mathrm{C}$. IR spectrum, $v, \mathrm{~cm}^{-1}: 1446,1467,1599,1660,2206(\mathrm{CN}), 3078-3325$ (NH). ${ }^{1} \mathrm{H}$ NMR spectrum $\left(\mathrm{CDCl}_{3}\right), \delta$, ppm: $1.86-1.93 \mathrm{~m}$ $\left(2 \mathrm{H}, \mathrm{CH}_{2}\right), 2.51 \mathrm{t}\left(2 \mathrm{H}, \mathrm{CH}_{2},{ }^{3} \mathrm{~J}_{\mathrm{HH}} 7.5 \mathrm{~Hz}\right), 2.79 \mathrm{~d}(3 \mathrm{H}$, $\left.\mathrm{CH}_{3},{ }^{3} \mathrm{~J}_{\mathrm{HH}} 6.5 \mathrm{~Hz}\right), 3.49 \mathrm{t}\left(2 \mathrm{H}, \mathrm{CH}_{2},{ }^{3} \mathrm{~J}_{\mathrm{HH}} 6.5 \mathrm{~Hz}\right), 5.05 \mathrm{br}$. $\mathrm{s}$ $(1 \mathrm{H}, \mathrm{NH}) .{ }^{13} \mathrm{C}$ NMR spectrum $\left(\mathrm{CDCl}_{3}\right), \delta_{\mathrm{C}}, \mathrm{ppm}: 23.3$, 27.5, 28.6, 42.2, $82.8\left(\mathrm{C}^{4}\right.$, oxazole), $114.4(\mathrm{CN}), 151.9$ ( $\mathrm{C}^{2}$, oxazole), $161.0\left(\mathrm{C}^{5}\right.$, oxazole). Mass spectrum, $m / z$ : $200[M+\mathrm{H}]^{+}$. Found, \%: C 48.32; H 5.28; Cl 17.57; N 21.21. $\mathrm{C}_{8} \mathrm{H}_{10} \mathrm{ClN}_{3} \mathrm{O}$. Calculated, \%: C 48.13; H 5.05; Cl 17.76; N 21.05.

4-Chloro- $N$-[cyano(imidazolidin-2-ylidene)methyl]butanamide (9). To a solution of $7.2 \mathrm{~g}(40 \mathrm{mmol})$ of ethylenediamine diacetate in $20 \mathrm{~mL}$ of methanol $2.4 \mathrm{~g}$ (10 mmol) was added in portions $(0.2 \mathrm{~g}$ each $)$ with vigorous stirring compound 3 at $20-25^{\circ} \mathrm{C}$. The reaction mixture was stirred for $48 \mathrm{~h}$, and ethylenediamide hydrochloride was filtered off. The solvent was removed in vacuum at $40^{\circ} \mathrm{C}$, then $20 \mathrm{~mL}$ of water was added to the residue, and the product was extracted with a mixture of $\mathrm{CH}_{2} \mathrm{Cl}_{2}$-propan-2-ol $(8: 2,6 \times 20 \mathrm{~mL})$. The solvent was removed in vacuum, the residue was treated with $20 \mathrm{~mL}$ of propan-2-ol, and the crystals were filtered off to give compound 9 as acetate. Yield $70 \%, \mathrm{mp} 61-63^{\circ} \mathrm{C}$. IR spectrum, $v, \mathrm{~cm}^{-1}: 1447,1475,1543,1601,1654,2207$ (CN), 3075-3417 (NH). ${ }^{1} \mathrm{H}$ NMR spectrum (DMSO- $d_{6}$ ), $\delta$, ppm: $1.79 \mathrm{~s}\left(3 \mathrm{H}, \mathrm{CH}_{3}\right), 2.01-2.10 \mathrm{~m}\left(2 \mathrm{H}, \mathrm{CH}_{2}\right), 2.71 \mathrm{t}$ $\left(2 \mathrm{H}, \mathrm{CH}_{2},{ }^{3} \mathrm{~J}_{\mathrm{HH}} 7.3 \mathrm{~Hz}\right), 2.85 \mathrm{t}\left(2 \mathrm{H}, \mathrm{CH}_{2},{ }^{3} \mathrm{~J}_{\mathrm{HH}} 6.0 \mathrm{~Hz}\right)$, $3.35 \mathrm{t}\left(2 \mathrm{H}, \mathrm{CH}_{2},{ }^{3} J_{\mathrm{HH}} 6.0 \mathrm{~Hz}\right), 3.70 \mathrm{t}\left(2 \mathrm{H}, \mathrm{CH}_{2},{ }^{3} \mathrm{~J}_{\mathrm{HH}}\right.$ $6.4 \mathrm{~Hz}), 6.65$ br. s $(4 \mathrm{H}, 3 \mathrm{NH}, \mathrm{OH}) .{ }^{13} \mathrm{C} \mathrm{NMR}$ spectrum $\left(\mathrm{DMSO}-d_{6}\right), \delta_{\mathrm{C}}$, ppm: 22.0, 23.2, 24.5, 27.8, $42.4\left(\mathrm{CH}_{2}\right)$, $43.3\left(\mathrm{CH}_{2}\right), 80.8\left[\underline{\mathrm{C}}=\mathrm{C}\left(\mathrm{NHCH}_{2}\right)_{2}\right], 115.7(\mathrm{CN}), 151.7$ $\left[\mathrm{C}=\underline{\mathrm{C}}\left(\mathrm{NHCH}_{2}\right)_{2}\right], 160.9(\mathrm{C}=\mathrm{O}), 174.0(\mathrm{C}=\mathrm{O})$. Mass spectrum, $m / z$ : $229\left[M+\mathrm{H}-\mathrm{CH}_{3} \mathrm{COOH}\right]^{+}$. Found, \%: C $45.99 ; \mathrm{H} 6.21 ; \mathrm{Cl} 12.20 ; \mathrm{N}$ 19.62. $\mathrm{C}_{11} \mathrm{H}_{17} \mathrm{ClN}_{4} \mathrm{O}_{3}$. Calculated, \%: C 45.76; H 5.93; Cl 12.28; N 19.40.

To obtain free base $9,2 \mathrm{~g}$ of acetate was dissolved in $5 \mathrm{~mL}$ of water, and then $5 \mathrm{~mL}$ of a saturated aqueous solution of $\mathrm{NaHCO}_{3}$ was added. The formed precipitate was filtered off, washed with water $(2 \times 3 \mathrm{~mL})$ and dried in vacuum at $40^{\circ} \mathrm{C}$. Yield $85 \%, \mathrm{mp} 80-82^{\circ} \mathrm{C}$. Mass spectrum, $m / z: 229[M+\mathrm{H}]^{+}$. Found, \%: C 47.04; H 5.70; $\mathrm{Cl} 15.79 ; \mathrm{N} 24.88 . \mathrm{C}_{9} \mathrm{H}_{13} \mathrm{ClN}_{4} \mathrm{O}$. Calculated, \%: C 47.27; H 5.73; Cl 15.50; N 24.50.

\section{AUTHOR INFORMATION}

O.V. Shablykin, ORCID: https://orcid.org/0000-00016810-9860

S.A. Chumachenko, ORCID: https://orcid.org/0000-00017020-8622

V.S. Brovarets, ORCID: https://orcid.org/0000-0001-66683412

\section{FUNDING}

This work was financially supported by the National Research Foundation of Ukraine (project no. 2020.01/0075).

\section{CONFLICT OF INTEREST}

No conflict of interest was declared by the authors.

\section{OPEN ACCESS}

This article is licensed under a Creative Commons Attribution 4.0 International License, which permits use, sharing, adaptation, distribution and reproduction in any medium or format, as long as you give appropriate credit to the original author(s) and the source, provide a link to the Creative Commons license, and indicate if changes were made. The images or other third party material in this article are included in the article's Creative Commons license, unless indicated otherwise in a credit line to the material. If material is not 
included in the article's Creative Commons license and your intended use is not permitted by statutory regulation or exceeds the permitted use, you will need to obtain permission directly from the copyright holder. To view a copy of this license, visit $\mathrm{http} / / /$ creativecommons.org/licenses/by/4.0/.

\section{REFERENCES}

1. Popil'nichenko, S.V., Brovarets, V.S., Chernega, A.N., Poltorak, D.V., and Drach, B.S., Heteroatom. Chem., 2006, vol. 17, no. 5, p. 411.

https://doi.org/10.1002/hc.20232

2. Kimura, H., Yukitake, H., Tajima, Y., Suzuki, H., Chikatsu, T., Morimoto, Sh., Funabashi, Y., Omae, H., Ito, T., Yoneda, Y., and Takizawa, M., Chem. Biol., 2010, vol. 17, no. 1, p. 18.

https://doi.org/10.1016/j.chembiol.2009.12.012

3. Chumachenko, S.A., Shablykin, O.V., Vasilenko, A.N., Rusanov, E.B., and Brovarets, V.S., Chem. Heterocycl. Compd., 2012, vol. 48, no. 6, p. 881. https://doi.org/10.1007/s10593-012-1070-7

4. Matsumura, K., Saraie, T., and Hashimoto, N., Chem. Pharm. Bull., 1976, vol. 24, no. 5, p. 924. https://doi.org/10.1248/cpb.24.924

5. Pil'o, S.G., Brovarets, V.S., Romanenko, E.A., and
Drach, B.S., Russ. J. Gen. Chem., 2002, vol. 72, no. 11, p. 1724.

https://doi.org/10.1023/A:1023385028068

6. Moskvina, V., Merzhyievskyi, D., Shablykin, O.V., Shablykin, O.V., Kozytskiy, A.V., Rusanov, E.B., and Brovarets, V.S., Eur. J. Org. Chem., 2021. https://doi.org/10.1002/ejoc.202100412

7. Drach, B.S. and Mis'kevich, G.N., J. Org. Chem. USSR, 1978, vol. 14, no. 3, p. 501.

8. Shablykin, O.V., Volosheniuk, M.A., and Brovarets, V.S., Russ. J. Gen. Chem., 2018, vol. 88, no. 7, p. 1542. https://doi.org/10.1134/S1070363218070307

9. Chumachenko, S.A., Shablykin, O.V., and Brovarets, V.S., Chem. Heterocycl. Compd., 2015, vol. 50, no. 12 , p. 1727.

https://doi.org/10.1007/s10593-015-1644-2

10. Matsumura, K., Saraie, T., and Hashimoto, N., Chem. Pharm. Bull., 1976, vol. 24, no. 5. P. 912. https://doi.org/10.1248/cpb.24.912

11. Klimova, V.A., Osnovnye mikrometody analiza organicheskikh soedinenii (Basic Micromethods for the Analysis of Organic Compounds), Moscow: Khimiya, 1975. 